\title{
Fútbol para personas con amputaciones: Revisión sistemática exploratoria Football for people with amputations: Exploratory systematic review
}

\author{
*José M. Gamonales, *M aykel Durán-Vaca, *Luisa Gámez-Calvo, *Víctor Hernández-Beltrán, * ,**jesús \\ Muñoz-Jiménez, ***Kiko León \\ *Universidad de Extremadura (España), **U niversidad Autónoma de Chile (Chile)
}

\begin{abstract}
Resumen. El trabajo tiene por objetivo revisar la evidencia científica relacionada con el fútbol para personas amputadas. Para la búsqueda de referencia, se utilizó como descriptores las siguientes palabras clave: «Football» y «Amputee». Los términos fueron introducidos en inglés en las siguientes bases de datos informatizadas: SPORT D iscus, PU BM ED,Web Of Science y SCOPUS, considerando todos los documentos publicados hasta septiembre del 2020. Para limitar la búsqueda, se introdujeron cuatro criterios de inclusión: 1) Seleccionar documentos disponibles a texto completo o con resumen, 2) Contar con suficiente información relevante sobre el fútbol para personas con amputaciones (Mínimo 50 palabras), 3) Aportar información relacionada con la condición física, entrenamiento, lesiones y/ o aspectos sociales de los jugadores de fútbol para per sonas con amputaciones, y 4) Estar escrito en español, ingléso portugués. La revisión sistemáticaexploratoriacontó con diez documentos relacionados con el fútbol para personas con amputaciones. Los documentos existentes relacionados con la temática son escasos como consecuencia de la fal ta de evolución de esta modal idad. Estosmanuscritos, en su mayoría, tratan aspectos como la fuerza y la resistencia. Además, un sólo documento habla de la utilización de dispositivos inerciales en sus mediciones. Por tanto, surge la necesidad de real izar nuevas investigaciones en el ámbito de fútbol para personas con amputaciones.
\end{abstract}

Palabras clave: Deporte adaptado, discapacidad, dispositivos inerciales.

\begin{abstract}
The study aims to review the scientific evidence related to football for amputees. For the reference search, the following keywords were used as descriptors: «Football» and «Amputee». The terms were entered in English in the following computerized databases: SPORTD iscus, PU BM ED, Web of Science and SCOPUS, considering all the documents published up to September 2020. To limit the search, four inclusion criteria were introduced: 1) Select documents available in full text or with abstract, 2) Have enough relevant information about soccer for amputees (M inimum 50 words), 3) Provide information related to the physical condition, training, injuries and / or social aspects of the players of soccer for people with amputations, and 4) Bewritten in Spanish, Engl ish or Portuguese. The exploratory systematic review featured ten football related documents for amputees. The existing documents related to the subject are scarce as a consequence of the lack of evolution of this modality. These manuscripts, for the most part, deal with aspects such as strength and endurance. Furthermore, only one document talks about the use of inertial devices in their measurements. Therefore, there is a need for further research in the field of football for amputees.
\end{abstract}

Key words: Adapted sport; disability; inertial devices.

\section{Introducción}

El fútbol es el deporte más extendido y practicado del mundo (Soler, 2009). Esta disciplina, tanto deportiva como recreativa, presenta beneficios físicos, sociales y psicológicos, además de servir como herramienta para educar en valores, establecer vínculos y promocionar estilos de vida y hábitos saludables (Lozano-Sufrategui, Pringle, Carless, \& M cKenna, 2017). Además, en el fútbol convencional, se hadesar rollado nuevas tecnologías que han permitido el progreso de diferentesdispositivospara el análisis de la carga externa e interna de los jugadores durante los entrenamientos y competiciones. Siendo,

Fecha recepción: 07-12-20. Fecha de aceptación: 15-03-21 José M artín Gamonales Puerto

josemartingamonales@gmail.com en los últimos 10 años, el Sistema de Posicionamiento Global, el medio más utilizado (Aytar, Seixas-da-Silva, Gomes de Souza \& De Alkmim, 2020; Izzo, DeVanna $\&$ Varde' i, 2018), con la finalidad de proporcionar estrategias de entrenamiento específicas (Casamichana \& Castellano, 2015), y programas de recuperación para reducir el número de lesiones (Drew \& Finch, 2016; M ohr, Krustrup \& Bangsbo, 2003). Por tanto, el fútbol es un deporte en constante perfeccionamiento, y una modalidad adaptable a cualquier colectivo independientemente de su género, edad o condición física (Tatar at al., 2018). De hecho, el fútbol es la práctica físico-deportiva que cuenta con más modalidades adaptadas.

Una de las modalidades de fútbol adaptado, más conocidas es el Fútbol a 7 para personas con Parálisis Cerebral (en adelante, Fútbol PC), organizada por la Federación Internacional de Fútbol PC (en adelante, 
IFCPF), bajo la supervisión de laA sociación Internacional para la Recreación y el Deporte de las Personas con Parálisis (Gamonales, León, Jiménez\& M uñoz-Jiménez, 2019). Los equipos están formados por siete jugadores y el juego se desarrolla en un terreno de juego al aire libre con unas dimensiones de 50 × 75 metros. Además, no existe el fuera de juego y los saques de banda pueden hacerse con una sola mano (IFCPF, 2020). Los jugadores, para poder competir, deben pasar por una Clasificación Funcional (en adelante, CF) previa, que ha evolucionado a lo largo de los años para permitir a cada deportista, independientemente de su discapacidad, competir deformajustacon el resto de losadversarios (Gorla et al., 2018; Reina, Elvira, Valverde, Roldán \& Yanci, 2019; Tweedy \& Vanlandewijck, 2011). Actualmente, los deportistas son clasificados en tres clases funciona les, CF1, CF2 y CF3 (Gamonales, 2020; Gamonales, Muñoz-Jiménez, Gómez-Carmona\& Ibáñez, 2021; Peña González, Sarabia, Mancha-Triguero, M oya-Ramón \& Gamonales, 2021).

0 tra modalidad adaptada muy practicada es el Fútbol a 5 para personas con discapacidad visual (en adelante, Fa5), siendo uno de los deportes de invasión de tanteo bajo más practicados por este colectivo (Gamonales, 2020; Gamonales, M uñoz-J iménez, León \& Ibáñez, 2018; Gamonales, Muñoz-Jiménez, León \& Ibáñez, 2019), y la única modalidad de fútbol para personas con discapacidad presente en los Juegos Paralímpicos. Sus normas se rigen por la FIFA, realizando adaptaciones para permitir la práctica a personas con discapacidad visual (Magno, M orato, Bilzon \& Duarte, 2013).

También, existe el fútbol para personas con discapacidad intelectual, una modalidad deportiva en pleno crecimiento, y que no presenta apenas adaptación en normativa. Los jugadores son clasificados funcional mente para jugar al fútbol en igualdad de condiciones, en función del grado de discapacidad. Esto es necesario porque las personas con discapacidad intelectual (en adelante, DI) pueden mostrar problemas de coordinación, y dificultades en la percepción, toma de decisiones y la atención, dificultando su participación en modalidades deportivas abiertas que requieren un alto nivel cognitivo (Abellán \& Sáez-Gallego, 2014). Los niveles de adaptación para los deportistas con DI son: nivel de competición, nivel adaptado y nivel de habilidades (Gamonales, 2020).

Respecto a las modalidades adaptadas para personas con unadiscapacidad física que requierasu desplazamiento en silla de ruedas son el Fútbol en Silla de Ruedas Eléctrica o Power Chair Football, creada en Francia en
1978 (Reis\& M ezzadri, 2017), y lamodalidad de Fútbol para Amputados (en adelante, FAm). El FAm es una modalidad deportiva cuyos participantes tienen alguna amputación de miembros superiores o inferiores (Auricchio et al., 2018; Kasiñska \& Tasiemski, 2017). Habitual mente, los jugadores tienen amputaciones unilaterales del miembro inferior, excepto el portero que su amputación se da en el miembro superior (W ieczorek, W iliñski, Struzik \& Rokita, 2015). Esta disciplina fue diseñada en 1982, con la finalidad de ser utilizada como entrenamiento para los esquiadores con discapacidades físicas (Simim et al., 2013), y se puede jugar en césped natural o sintético con un mínimo de 60 $x 30$ metrosy un máximo de 70 x 55 metros. El equipo está formado por siete jugadores y un portero. El partido se subdivide en dos tiempos de veinticinco minutos, con un descanso de diez minutos entre ellos. El FAm implica actividad explosiva como saltos, patadas, cambios de dirección, sprints de al ta velocidad y control del balón mientras se esquiva a los oponentes (Aytar, Pekyavas, Ergun \& Karatas, 2012).

Por último, en la literatura científica, existen estudios relacionados con el fútbol convencional y la recogida de datos con dispositivos inerciales para controlar las cargas de entrenamiento (Díaz, Bradley, Díaz \& Pallarés, 2013), y conocer los datos reales de los partidos (Aytar et al., 2020; Dios, Jiménez, García \& García, 2018). Además, existen trabajos relacionados con deportes para personas con discapacidad, como en Fa5 donde se anal iza la carga interna y externa de los jugadores durante un torneo internacional (Gamonales, Muñoz-Jiménez, Mancha-Triguero \& Ibáñez, 2020), y en Fa7PC, donde se analiza la carga externa en función de la clasificación funcional de los deportistas (Gamonales et al., 2021; Peña-González et al. , 2021; Reina, Iturricastillo, CastiIlo, U rban, \&Yanci, 2019). Por tanto, sería recomenda ble ampliar el conocimiento del FAm, de forma que los investigadores utilicen los dispositivos inerciales para recoger los datos de los jugadores durante los entrenamientos y partidos, así como para la realización de pruebas específicas, con la final idad de conocer laFC máx, Player Load, Velocidades a diferentes intensidades, aceleraciones y desaceleraciones. Además, hay poca litera tura específica que aborde el FAm. Por ello, los objetivos de este estudio son: a) realizar una revisión sistemática exploratoria relacionada con el FAm, b) analizar las capacidades físicas básicas implicadas en los jugadores de FAm, y c) conocer si los autores de los documentos seleccionados en la revisión utilizan dispositivos inerciales como herramienta novedosa para cuantificar 
la carga interna y externa durante los entrenamientos y partidos.

\section{M étodo}

\section{Diseño}

Esta investigación se centra dentro de los estudios teóricos. Para ello, se realizó una búsqueda y recopila ción de documentos científicos mediante un modelo de «Acumulación de datos y sel ección de estudios» (Ato, López $\&$ Benavente, 2013), con el objetivo de analizar los documentos relacionados con el FAm.

\section{Muestra}

La muestra estuvo compuesta por diferentes documentos científicos $(n=10)$, relacionados con el FAm, y el conocimiento del empleo de dispositivos inerciales durante los entrenamientos y partidos. En la primera búsqueda, se utilizó el término «Football», y se encontraron un total de 375.870 manuscritos, de los cuales se rechazaron 375.769 , tras introducir el segundo término «Amputee». Es decir, a medida que se iban introduciendo las palabras claves en las bases de datos, el número de documentos se iban reduciendo. Posteriormente, se aplicaron los Criterios para la selección de los estudios, y se seleccionaron 10 manuscritos científicos relacionados con el FAm.

\section{Criterios para la selección de los estudios}

Para la búsqueda y selección de documentos, se introdujeron las palabras clave, en inglés: «Football» y "Amputee», en las bases de datos empleadas computarizadas: Web of Science (en adelante, WOS), PUBMED, SPORTD Iscus y SCOPUS. Los manuscritos seleccionados para formar parte de la muestra debían de cumplir una serie de criterios de inclusión: 1) Seleccionar documentos disponibles a texto completo o con resumen, 2) Contar con suficiente información relevante sobre el fútbol para personas con amputaciones (Mínimo 50 palabras), 3) Aportar información relacionada con la condición física, entrenamiento, lesiones y/ 0 aspectos social es de los jugadores de fútbol para personas con amputaciones, y 4) Estar escrito en español, inglés o portugués. Además, se emplearon criterios de exclusión de manuscritos con la finalidad de determinar que documentos no son válidosy así poder extraer informa ción relevante relacionada con el FAm: 5) Eliminar los documentos en los que solamente se mencionen la/ $\mathrm{s}$ palabra/ sclave/ sintroducida/ sen la base datos, 6) Excluir los manuscritos que no se puedan referenciar, 7)
Suprimir los documentos relacionados con otras modalidades de fútbol adaptado, y 8) Excluir los manuscritos que se refieren al fútbol en general.

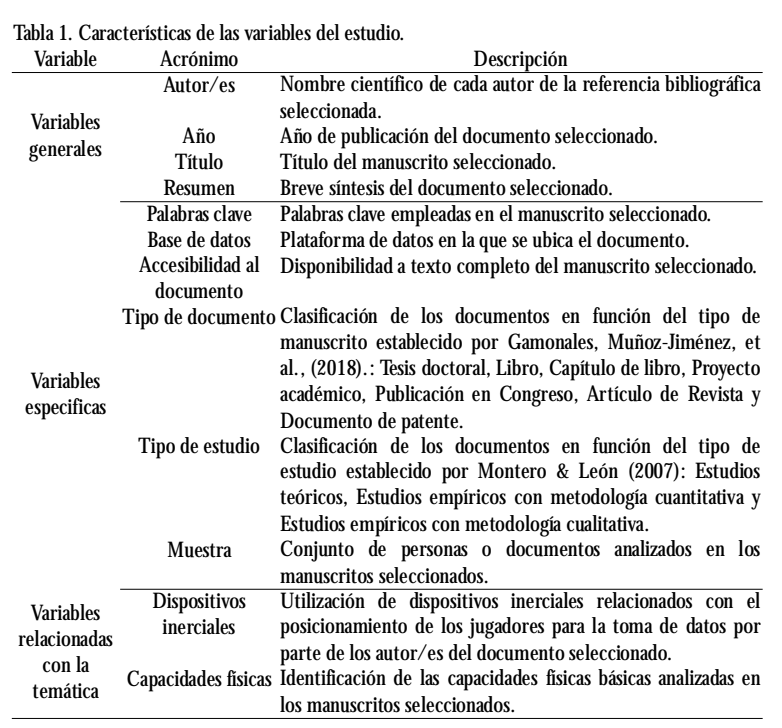

\section{Codificación de las variables}

En laTabla 1, se muestran las variables del estudio de revisión sistemática exploratoria en FAm: Variables generales (Autor/ es, Año,Título y Resumen), y Variables específicas relacionadas con los documentos seleccionados ( $\mathrm{Pa}$ labras clave, Base de datos, Accesibilidad al documento, Tipo de documento, Tipo de estudio, M uestra), así como Variables relacionada con la temática deinvestigación (D ispositivos Inerciales y Capacidades Físicas).

\section{Procedimiento de registro para los estudios}

El procedimiento utilizado en este trabajo es similar a los existentes en la literatura científica ( $G$ ámezCalvo, Gamonales, Silva-O rtiz \& M uñoz-Jiménez 2020; Gamonales, Muñoz-Jiménez et al., 2018; Silva-O rtiz, Gamonales, Gámez-Calvo, \& Muñoz-Jiménez, 2020). Para ello, es fundamental realizar una adecuada planificación de la búsqueda literaria con la finalidad de favorecer su éxito (Thomas, Silverman \& Nelson, 2015), y extraer conclusiones relevantes (Gamonales, GilSánchez et al., 2018). Todos los documentos seleccionados para el estudio cumplían con los criterios de inclusión establecidos. En la Figura 1, se muestran las fases del proceso de búsqueda llevado a cabo para el desarro-

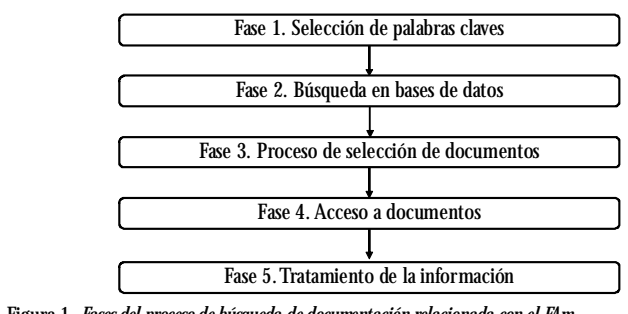

Figura 1. Fases del proceso de búsqueda de documentación relacionada con el FAm. 
Ilo de este trabajo, con la finalidad de mejorar la compresión lectora.

Fase 1. Planificación y selección de palabras clave para la búsqueda. Para seleccionar las palabras clave, se tuvo en cuenta los objetivos de la revisión sistemática exploratoria relacionada con FAm y el empleo de dispositivos inerciales. Para localizar la mayor cantidad de documentos científicos, se realizó la búsqueda de pala bras clave en inglés, utilizando «Football» y «Amputee». Para ello, se establecieron los criterios de inclusión y exclusión mencionados anteriormente. En todas las ba ses de datos consultadas, se emplearon las mismas pala bras clave.

Fase 2. Búsqueda en bases de datos. Se realizaron cuatro búsquedas bibliográficas en las siguientes bases de datos informatizadas WOS, PUBMED, SPORTDiscus y SCOPUS. Para ello, se empleó el mismo procedimiento booleano (And) de búsqueda en las diferentes plataformas de da tos, con el propósito de encontrar la mayor cantidad posible de documentos relacionados. La frase de búsqueda final fue: Football - And - Amputee. En la Figura2, se muestra el procedimiento de búsqueda, y el número de documentos existentes en función de las palabras cla ves introducidas. A medida que se iban introduciendo los términos claves en el buscador, estos resultados iban disminuyendo considerablemente. Posteriormente, se aplicaron los criterios de inclusión y exclusión de ma nuscritos.

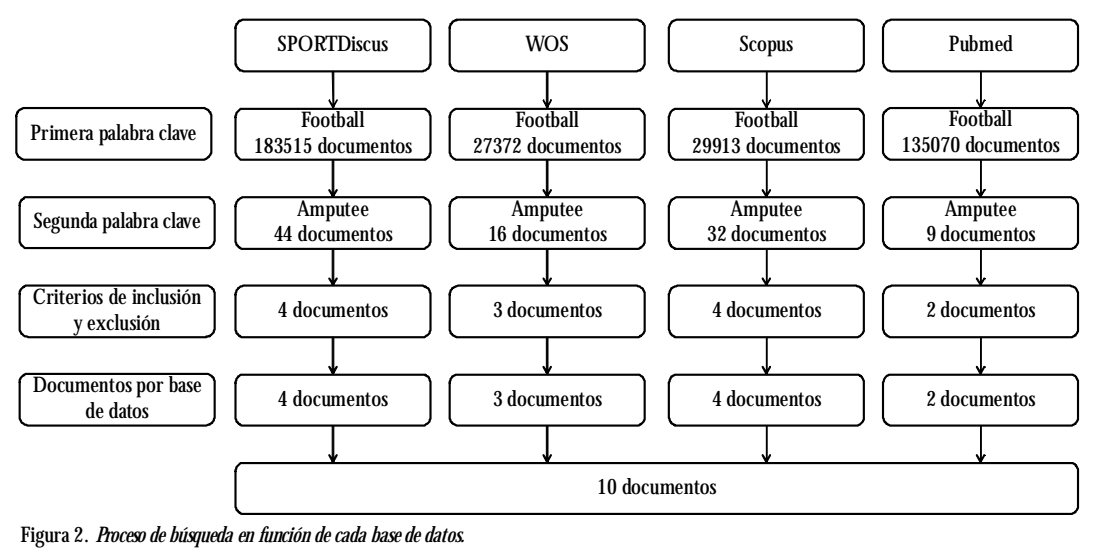

Fase 3. Proceso de selección de documentos. Tras introducir las distintas palabras claves en las diferentes bases de datos, se procedió a la selección de documentos. Para ello, fue importante establecer unos criterios de inclusión y exclusión. Este procedimiento, se llevó a cabo para rechazar aquellos manuscritos que no cumplían con los mínimos exigidos por los investigadores.

Fase 4. Acceso a documentos. Algunas de las bases de datos utilizadas para la búsqueda de referencias no permiten acceso a documentos a texto completo. Por tan- to, para poder consultar la mayor cantidad de trabajos originales a texto completo se empleó el portal web de la biblioteca electrónica de la Universidad de Extremadura, así como distintas plataformas y buscadores web de contacto con los autores como realizaron los autores Gamonales, Muñoz-J iménez, et al., (2018). La muestra quedó reducida a 10 manuscritos, que fueron revisados en profundidad por los autores del documento de la revisión sistemática exploratoria relacionada con el FAm. Además, el número de documentos elegidos no debe ser muy numeroso, puesto que, el tratamiento de la información podría ser contaminado por el investigador (Benito-Peinado et al., 2007).

Fase 5. Tratamiento de la información. De cada documento seleccionado, se analizaron las Variables generales, Variables específicas y Variables relacionada con la temática de investigación. Por este motivo, es importante la organización y categorización de toda la información obtenida. Además, se realizó una síntesis de cada manuscrito, mediante la elaboración de una tabla con la toda información que permitiera su revisión de manera óptima y eficaz. Se realizó un análisis descriptivo y exploratorio de las Palabras Clave, Base de datos, Idioma, Dispositivos inerciales, y Capacidades físicas de los diferentes manuscritos con la finalidad de conocer su vinculación con la temática del estudio (Gamonales, M uñoz-Jiménez et al., 2018).

\section{Análisis estadístico}

Se real izó una anál isis descriptivo y exploratorio relacionado con las variables: Palabras Clave, Base de datos, Tipo de documento, Tipo deestudio, Dispositivos inerciales, y Capacidadesfísicas, con el objetivo de aportar información relevante y visual paralos lectores, así como dar acla raciones significativas relacionadas con los documentos seleccionados. Para el análisis estadístico, se utilizó el softwareStatistical Package of Social Science (versión 24, 2016; IBM Corp. IBM SPSSStatisticsparaMAC OS, Armonk, NY, EEUU).

\section{Resultados}

En laTabla 2, se muestran los documentos seleccionados relacionados con el FAm, y el empleo de dispositivos inerciales como herramienta novedosa para cuantificar la carga interna y externa durante los entrena 
Tabla 2. Documentos seleccionados para la revisión sistemática exploratoria en relación con el FAm.

\begin{tabular}{|c|c|c|c|c|c|c|c|c|c|c|}
\hline & Autor/ es Año & Título & Resumen & PC & $\mathrm{BD}$ & Muestra & & DI & TD TE & Cap. Físicas \\
\hline & Tatar et al. & $\begin{array}{l}\text { Load distribution on the foot } \\
\text { and lofstrand crutches of } \\
\text { amputee football players. }\end{array}$ & $\begin{array}{l}\text { Se calculó la distribución de la carga en jugadores de FAm al real izar } \\
\text { tres acciones básicas: caminar, correr y lanzar a portería. Se } \\
\text { comprueba que durante la fase de gol peo la carga es mayor para el } \\
\text { miembro superior, mientras que durante la carrera y caminata la } \\
\text { carga es mayor para miembros inferiores. }\end{array}$ & $\begin{array}{l}\text { Amputee Football, } \\
\text { Lofstrand Crutche, } \\
\text { Loading, Kicking }\end{array}$ & $\begin{array}{l}\text { SD, } \\
\text { PubMed, } \\
\text { WOS, } \\
\text { Scopus }\end{array}$ & 25 jugadores & $\mathrm{Si}$ & No & $\begin{array}{l}\text { PA Emp. } \\
\text { Cuant. }\end{array}$ & $\begin{array}{l}\text { Resistencia, } \\
\text { velocidad y } \\
\text { fuerza. }\end{array}$ \\
\hline 2 & Simim et al. 2018 & $\begin{array}{l}\text { The Demands of Amputee } \\
\text { Soccer Impair M uscular } \\
\text { Endurance and Power Indices } \\
\text { but not match Physical } \\
\text { Performance. }\end{array}$ & $\begin{array}{l}\text { El objetivo del estudio fue conocer las demandas físicas de los } \\
\text { jugadores de FAm durante un partido. Los resultados muestran que } \\
\text { la frecuencia cardíaca y la velocidad máxima no se diferencia en } \\
\text { ambas mitades, mientras que las medidas de rendimiento } \\
\text { neuromuscular disminuyeron tras las mediciones después del } \\
\text { partido. }\end{array}$ & $\begin{array}{l}\text { Disability Sport, } \\
\text { Fatigue, Football, } \\
\text { Match Analysis. }\end{array}$ & $\begin{array}{l}\text { PubMed, } \\
\text { WOS, } \\
\text { Scopus }\end{array}$ & d, 16 jugadores & No & No & $\begin{array}{l}\text { AR Emp. } \\
\text { Cuant. }\end{array}$ & $\begin{array}{l}\text { Resistencia, } \\
\text { velocidad y } \\
\text { fuerza. }\end{array}$ \\
\hline ( & Mikami et al. 2018 & $\begin{array}{l}\text { Exercise loading for } \\
\text { cardiopulmonary assessment } \\
\text { and evaluation of endurance in } \\
\text { amputee football players. }\end{array}$ & $\begin{array}{l}\text { Se real izaron pruebas cardiopulmonares de tres tipos: con dos } \\
\text { brazos, con una pierna y con dos pier nas en adultos sanos, con el } \\
\text { nobjetivo de comparar los resultados de estas, y también con } \\
\text { jugadores de FAm. Se comprobó que los factores músculo } \\
\text { esqueléticos tienen mayor importancia para las pruebas de una pierna } \\
\text { que para la de dos brazos. }\end{array}$ & $\begin{array}{l}\text { Amputee Football, } \\
\text { Endurance, } \\
\text { Cardiopulmonary } \\
\text { Exercise Test } \\
\text { a }\end{array}$ & PubMed & $\begin{array}{l}20 \text { jugadores (25- } \\
30 \text { años) }\end{array}$ & Si & No & $\begin{array}{l}\text { AR Emp. } \\
\text { Cuant. }\end{array}$ & $\begin{array}{l}\text { Fuerza y } \\
\text { resistencia. }\end{array}$ \\
\hline & $\begin{array}{l}\text { Yildiz, } 2018 \\
\text { Kayihan, } \\
\text { Gelen, Cilli \& } \\
\text { Ozkan }\end{array}$ & $\begin{array}{l}\text { Acute effects of different } \\
\text { warm-up methods on free } \\
\text { kick performance in amputee } \\
\text { soccer players. }\end{array}$ & $\begin{array}{l}\text { Se compara el efecto de tres métodos de calentamiento (estiramiento } \\
\text { estático, dinámico y control) en el rendimiento del tiro libre en } \\
\text { futbolistas amputados. Para ello, se mide con una pistola que mide la } \\
\text { velocidad del objeto, y se comprueba los efectos positivos para el } \\
\text { estiramiento dinámico previo al lanzamiento, y negativos para el } \\
\text { estiramiento estático. }\end{array}$ & $\begin{array}{l}\text { oAmputees, Football, } \\
\text { Muscle, Stretching } \\
\text { Exercises, Warm-Up } \\
\text { Exercise. }\end{array}$ & $\begin{array}{l}\text { WOS, } \\
\text { Scopus }\end{array}$ & 12 jugadores & $\mathrm{Si}$ & No & $\begin{array}{l}\text { AR Emp. } \\
\text { Cuant. }\end{array}$ & $\begin{array}{l}\text { Flexibilidad } \\
\text { y velocidad. }\end{array}$ \\
\hline 5 & $\begin{array}{l}\text { Guchan, } 2017 \\
\text { Bayramlar \& } \\
\text { Ergun }\end{array}$ & $\begin{array}{l}\text { Determination of the effects } \\
\text { of playing soccer on physical } \\
\text { fitness in individuals with } \\
\text { transtibial amputation. }\end{array}$ & $\begin{array}{l}\text { El objetivo del estudio fue deter minar los efectos en componentes } \\
\text { físicos, como la composición corporal o la potencia de los jugadores } \\
\text { de FAm. Se añadió un grupo control (amputados), y se obtuvieron } \\
\text { mejores resultados en flexibilidad, porcentaje graso y potencia en } \\
\text { relación con el grupo de jugadores de fútbol convencional. }\end{array}$ & $\begin{array}{l}\text { Physical Fitness, } \\
\text { Amputees, Soccer, } \\
\text { Disabled Persons, } \\
\text { Athletes. }\end{array}$ & $\begin{array}{l}\text { PubMed, } \\
\text { Scopus }\end{array}$ & $\begin{array}{l}\text {, } 12 \text { jugadores (26- } \\
32 \text { años) }\end{array}$ & No & No & $\begin{array}{l}\text { AR Emp. } \\
\text { Cuant. }\end{array}$ & $\begin{array}{l}\text { Resistencia, } \\
\text { vel ocidad y } \\
\text { fuerza. }\end{array}$ \\
\hline 6 & Simim et al. 2017 & $\begin{array}{l}\text { The quantification of game- } \\
\text { induced muscle fatigue in } \\
\text { amputee soccer players. }\end{array}$ & $\begin{array}{l}\text { Se anal izó la fatiga de los jugadores de FAm tras un partido, con tres } \\
\text { actividades: empuje, salto con contra movimiento y lanzamiento con } \\
\text { balón medicinal. Se comprobó un descenso del rendimiento tras el } \\
\text { partido para estas actividades, y más pronunciado en la actividad de } \\
\text { empuje. }\end{array}$ & $\begin{array}{l}\text { Exercise, Sports for } \\
\text { Persons with } \\
\text { Disabilities, Muscle } \\
\text { Strength. }\end{array}$ & WOS & 33 jugadores & No & No & $\begin{array}{l}\text { AR Emp. } \\
\text { Cuant. }\end{array}$ & Fuerza \\
\hline 7 & $\begin{array}{l}\text { Kasinska \& } \\
\text { Tasiemski }\end{array}$ & $\begin{array}{l}\text { Amputee football in practice } \\
\text { and research. }\end{array}$ & $\begin{array}{l}\text { El objetivo del estudio es realizar una revisión del FAm desde su } \\
\text { historia hasta las investigaciones científicas realizadas sobre el mismo. } \\
\text { Para ello, anal izan el origen de la modalidad en Polonia, y muestra } \\
\text { toda la trayectoria de este deporte. Se basa en once artículos } \\
\text { divididos en tres conceptos: aspectos sociales, antropometría y } \\
\text { nutrición, y condición física. }\end{array}$ & $\begin{array}{l}\text { LimbAmputations, } \\
\text { Crutch Football, } \\
\text { Sport for People } \\
\text { with Disabilities. }\end{array}$ & Scopus & 11 documentos & Si & No & AR Est.T & $\begin{array}{l}\text { Resistencia, } \\
\text { vel ocidad y } \\
\text { fuerza. }\end{array}$ \\
\hline 8 & $\begin{array}{l}\text { W ieczorek et } 2015 \\
\text { al. }\end{array}$ & $\begin{array}{l}\text { Hand grip strength vs. sprint } \\
\text { effectiveness in amputee } \\
\text { soccer players. }\end{array}$ & $\begin{array}{l}\text { Se anal iza la relación de la fuerza de agarre de la mano y el tiempo de } \\
\text { carrera en FAm. La fuerza de agar re se midió con dinamómetro y el } \\
\text { tiempo de sprint con sensores cada } 5 \mathrm{~m} \text { en línea recta de } 30 \mathrm{~m} \text {. No se } \\
\text { identifican relaciones significativas entre la fuerza de agarre (derecha } \\
\text { o izquierda), y el tiempo de sprint en } 30 \mathrm{~m} \text {. }\end{array}$ & $\begin{array}{l}\text { eAmputeeAthletes, } \\
\text { RunningVelocity, } \\
\text { Soccer. }\end{array}$ & WOS & 12 jugadores & $\mathrm{Si}$ & No & $\begin{array}{l}\text { AR Emp. } \\
\text { Cuant. }\end{array}$ & $\begin{array}{l}\text { Velocidad y } \\
\text { fuerza. }\end{array}$ \\
\hline$\overline{9}$ & Simim et al. 2013 & $\begin{array}{l}\text { Anthropometric profile and } \\
\text { physical performance } \\
\text { characteristic of the Brazilian } \\
\text { amputee football (soccer) } \\
\text { team. }\end{array}$ & $\begin{array}{l}\text { Se anal izan las características antropométricas, l a frecuencia cardiaca } \\
\text { y distancia recorrida en jugadores de FAm. Para las medidas } \\
\text { antropométricas, se anal lizaron la masa cor poral, la grasa corporal y la } \\
\text { estatura, y para la frecuencia cardiaca, se realizaron dos pruebas } \\
\text { submáximas. El porcentaje graso fue diferente para mediocampistas } \\
\text { que, para el resto de las posiciones, y en las pruebas máximas } \\
\text { obtuvieron resultados inferiores a cualquier ecuación predictiva. }\end{array}$ & $\begin{array}{l}\text { Adapted Physical } \\
\text { Activity, } \\
\text { laAmputation, Per sons } \\
\text { with Disability. }\end{array}$ & Scopus & 12 jugadores & No & $\mathrm{Si}$ & $\begin{array}{l}\text { AR Emp. } \\
\text { Cuant. }\end{array}$ & Resistencia \\
\hline & $\begin{array}{l}\text { Yazicioglu, } 2007 \\
\text { Taskaynatan, } \\
\text { Guzelkucuk } \\
\text { \& Tugcu }\end{array}$ & $\begin{array}{l}\text { Effect of playing football } \\
\text { (soccer) on balance, strength, } \\
\text { and quality of life in unilateral } \\
\text { below-knee amputees. }\end{array}$ & $\begin{array}{l}\text { Se anal izó los efectos del FAm, en personas amputadas del miembro } \\
\text { inferior unilateralmente, sobre el equilibrio, la fuerza y calidad de } \\
\text { I vida. El fútbol tiene efectos positivos en el equilibrio y cal idad de } \\
\text { vida para personas amputadas. }\end{array}$ & $\begin{array}{l}\text { Football, Q uality of } \\
\text { Life, Amputees. }\end{array}$ & $\begin{array}{l}\text { PubMed, } \\
\text { WOS, } \\
\text { Scopus }\end{array}$ & $\begin{array}{l}\text { G G rupo de estudio } \\
\text { y grupo de control } \\
\text { (3 días) }\end{array}$ & & No & $\begin{array}{l}\text { AR Emp. } \\
\text { Cuant. }\end{array}$ & Fuerza \\
\hline
\end{tabular}

mientosy partidos, así como las principales capacidades físicas analizadas en cada estudio. Por otro lado, se muestran las diferentes Palabras claves utilizadas en cada documento seleccionado. Los vocablos que se repiten un

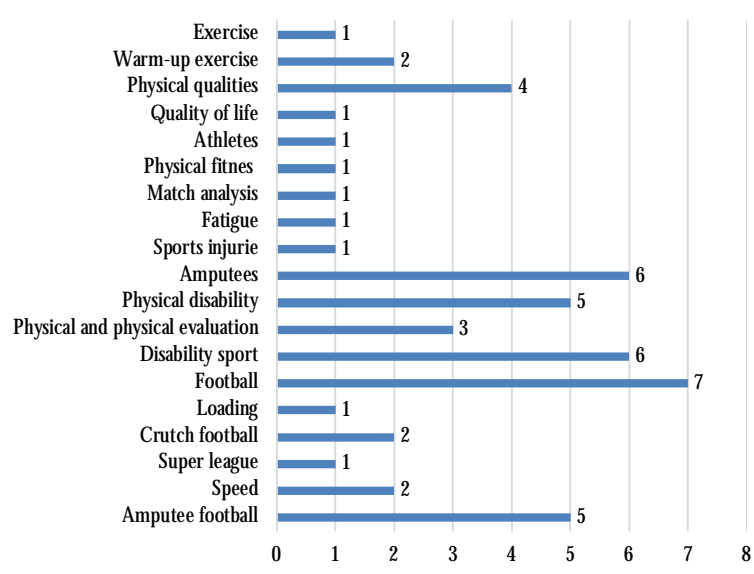

Figura 3. Análisis de los documentos seleccionados en función de la Pal abras clave. mayor número de veces son: Football $(n=7)$, Disability sport $(n=6)$, Amputees $(n=6)$, y Amputee football $(n=5)$. Respecto a las Bases de datos, en la plataformaSCOPUS se encontraron siete documentos $(n=7)$. En el resto de las bases de datos, el número de manuscritos seleccionado fue descendiendo progresivamente, en la WOS $(n=6)$, PUBMED $(n=5)$, y SPORTDiscus $(n=4)$. Algunos de los documentos coincidían en varias bases de datos. LOS documentos fueron seleccionados de acuerdo con los criterios de inclusión y exclusión establecidos. Respecto al Tipo de estudio empleado por los autores de los documentos seleccionados, predominan los manuscritos de análisis Empíricos cuantitativos $(n=11)$. Por último, se muestran las Capacidades físicas analizadas en los diferentes documentos seleccionados relacionados con el FAm. Siendo, la Velocidad $(n=6)$, y la Fuerza $(n=7)$, las capacidades físicas que más se trabaja en FAm durante los entrenamientos. 


\section{Discusión}

El objetivo de este trabajo fue realizar una revisión sistemática exploratoria relacionada con del FAm, y analizar los documentos seleccionados con la finalidad de conocer si los propios autores de los manuscritos emplean en sus investigaciones dispositivos inerciales como herramienta novedades en las Ciencias del Deporte, así como conocer qué capacidades físicas son las que se trabajan primordialmente durante los entrena mientos. El número de documentos relacionados con la temática existentes en las bases de datos son escasos, estando, la mayoría relacionados con las demandas físicas. Además, el procedimiento utilizado en la revisión sistemática exploratoria de los documentos ha seguido procesos similares a los existentes en la literatura (Gámez-Calvo et al., 2020; Gamonales, Gil-Sánchez et al., 2018; Gamonales, Muñoz-Jiménez et al., 2018), con la finalidad de seleccionar los manuscritos más adecua dos a la temática objeto de estudio.

En cuanto a las Palabras Claves, los vocablos principa les son: Football $(n=7)$, Disability sport $(n=6)$, Amputees $(n=6)$ y Amputee football $(n=5)$. En la literatura científica, no existen estudios que corroboren los resultados obtenidos. Sin embargo, los términos extraídos están vinculados con la temática objeto de estudio. Además, hay que mencionar que dicho análisis estadístico fue determinante para establecer las palabras clave del documento de revisión sistemática exploratoria en FAm, como sugieren los autores $G$ amonales, Muñoz-Jiménez et al., (2018), en su estudio de la literatura en Fa5.

Respecto a los documentos seleccionados en FAm, se han encontrado diferentes manuscritos en todas las bases de datos utilizadas. Siendo SCOPUS, la base de datos que presenta un mayor número de documentos $(n=7)$, relacionados con la temática. En la literatura científica, los estudios relacionados con el Fa5 predominan en labasededatosWOS (Gamonales, M uñoz-jiménez et al., 2018). Por tanto, hay que indicar que el número de manuscritos variará en función de la temática, y de las indexaciones de las revistas. La única base de datos especifica de contenido deportivo utilizada en esta revisión ha sido SPORTD iscus, la cual, presentó un número menor de documentos que las bases de datos PubM ed y WOS. Por ello, se recomienda a los investigadores en Deporte y Discapacidad publicar en revistas deportivas indexadas con el objetivo de facilitar la búsqueda de documentos, con la finalidad de dar mayor visualidad a los trabajos en Ciencias del Deporte.

En la literatura científica, no existen estudios que corroboren los resultados obtenidos. Sin embargo, hay que indicar que se necesita aumentar el número de contribuciones científicas relacionadas con el uso dispositivos inerciales para analizar el rendimiento en FAm, con la publicación de Tesis doctorales, Libros, Capítulos de libros, Proyectos académicos, Publicaciones en congresos y/ 0 reuniones científicas, Artículos de revista y Documentos de patentes, con la finalidad de ampliar el conocimiento en dicha disciplina.

En cuanto a los documentos seleccionados que utilizan dispositivos inerciales en sus estudios para registrar la carga interna y externa de los jugadores de FAm durante los entrenamientos y partidos, son escasos (10\% ). El único documento en el que se utiliza dispositivos inerciales es el realizado por Simim et al., (2013), en el que son empleados para medir las distancias recorridas y la frecuencia cardiaca.

Por último, los resultados relacionados con las Capacidades Físcas muestran que la Fuerza $(n=8)$, la Resistencia $(n=6)$, y la Velocidad $(n=6)$, son las cualidades más analizadas por los investigadores de los documentos seleccionados. Es decir, son capacidades físicas importantes en el rendimiento en fútbol para personas con amputaciones. Sin embargo, en los últimos años, se está dando gran importancia en el fútbol convencional a la Flexibilidad, contando con tan solo un estudio en el que se analiza esta capacidad física, con la finalidad de prevenir lesiones en los jugadores (Villarejo, Belmonte, Cejudo \& Elvira, 2019). Respecto al FAm, el trabajo de Flexibilidad se realiza con estiramientos estáticos, diná micos y de control, en los que se observan efectos positivos para el calentamiento dinámico previo al lanzamiento a portería (Yildiz et al., 2018). Además, resulta útil trabajar la Resistencia, con lafinalidad de aguantar los partidos completos (Simim et al., 2018), y puede valorarse con el empleo de diferentes pruebas de resistencia (Simim et al., 2013). Por otro lado, en los documentosseleccionados paralarevisión sistemáticaexploratoria en FAm, la Fuerza es evaluada de distintas maneras, a través de actividades de empuje y lanzamiento (Simim et al., 2017), y agarres medidos con dinamómetros (W ieczorek et al., 2015). Por último, en los manuscritos seleccionados, la Velocidad es analizada de dos formas por medición del tiempo de reacción del jugador 0 mediante el tiempo que tarda en recorrer 30 metros (W ieczorek et al., 2015). En la literatura existente, los documentos relacionados con las capacidades físicas en los jugadores de fútbol para personas con discapacidad son escasos, siendo el fútbol PC, lamodalidad que cuenta con un mayor número de publicaciones científicas. 


\section{Conclusiones}

El presente documento se postula como la primera revisión de habla hispana relacionada con el FAm, y recoge información acerca de las capacidades físicas básicas implicadas en los jugadores de FAm.

Los documentos existentes relacionados con el FAm son escasos, produciendo como consecuencia falta de evolución de esta modalidad deportivay su no inclusión en los Juegos Paralímpicos.

Las investigaciones relacionadas con el FAm no utilizan dispositivos inerciales para la recogida de datos, siendo estos instrumentos una herramienta esencial en el fútbol convencional.

El FAm es una modalidad que se encuentra en pleno auge, tanto a nivel nacional como internacional, siendo una de las modalidades deportivas más importantes para las personas con discapacidad física. Los estudios actuales en torno al FAm analizan diferentes capacidades físicas, como la fuerza, la velocidad y la resistencia, como factores determinantes del rendimiento, pero ningún estudio concluye cual de estas capacidades es más importante para conseguir un rendimiento óptimo. Por tanto, se recomienda la realización de futuros proyectos en este ámbito, con la utilización de dispositivos inerciales para la recogida de datos.

\section{Agradecimientos y Financiación}

Trabajo desarrollado dentro del Grupo de 0 ptimización del Entrenamiento y Rendimiento Deportivo (GOERD) de la Facultad de Ciencias del Deporte de la U niversidad de Extremadura. Además, este trabajo ha sido parcialmente subvencionado por laAyudaalos Gruposde Investigación (GR18170), de laJunta de Extremadura (Consejería de Empleo e Infraestructuras); con la aportación de la U nión Europea a través de los Fondos Europeos de Desarrollo Regional (FEDER).

\section{Referencias}

A bellán, J., \& Sáez-Gallego, N.M. (2014). Justificación de las pruebas motrices en el deporte para personas con discapacidad intelectual. Revista Iberoamericana de Psicología del Ejercicio y el Deporte, 9(1), 143-153.

Ato, M., López, J... \& Benavente, A. (2013). Un sistema de clasificación de los diseños de investigación en psicología. Anales de Psicología, 29(3), 1038-1059. https:/ / dx.doi.org/ 10.6018/ analesps.29.3.178511
Auricchio, J.R., Bernardes, N., Luksevicius-Rica, R., Quintas de Oliveira, R.C., Bocalini, D.S., \& Aparecida-M oreno, M. (2018). Avaliação antropométrica, hemodinâmica e metabólica da Seleção Brasileira de Futebol de Amputados. Motricidade, 14(1), 289-293.

Aytar, A., Pekyavas, N. O., Ergun, N., \& Karatas, M. (2012). Is there a relationship between core stability, balance and strength in amputee soccer players? A pilot study. Prosheticsand Orthotics International, 36(3), 332-338.

Aytar, A., Seixas-da-Silva, I. A., Gomes de Souza, R., \& De Alkmim, R. (2020). Tecnología GPS para controlar las demandas externas de las jugadoras de fútbol brasileñas de élite durante las competiciones. RETOS: N uevasTendencias en Educación Física, Deportes y Recreación, 40(2), 18-26. https:/ I doi.org/ 10.47197/ retos. v1i40.81943

Benito-Peinado, P.., Díaz-M olina,V., Calderón-M ontero, F.J., Peinado-Lozano, A.B., M artín-Caro, C., Álvarez-Sánchez, M., \& Pérez-Tejero, J. (2007). La revisión bibliográfica sistemática en fisiología del ejercicio: recomendaciones prácticas. Revista Internacional de Ciencias del Deporte, 3(6), 1-11. https:/ / doi.org/ doi:10.5232/ ricyde2007.00601

Casamichana, D., \& Castellano, J. (2015). The relationship between intensity indicators in smallsided soccer games. Journal of H uman Kinetics, 45, 119-128. https:/ / doi.org/ 10.1515/ hukin-2015-0040

Díaz,A.J., Bradley, P.S., Dízz, A., \& Pallarés, J.G. (2013). Percepción subjetiva del esfuerzo en fútbol profesional: relevancia de los indicadores físicos y psicológicos en el entrenamiento y la competición. Anales de Psicología, 29(3), 656-661.

Dios, R.M., Jiménez, M.A., García, J.E., \& García, S. L. (2018). Análisis observacional de las relaciones interlíneas de la Selección Española de Fútbol, mediante coordenadas polares. Cuadernos de Psicología del Deporte, 18(2), 18-32.

Drew, K., \& Finch, C.F. (2016). Relationship between training load and injury, illness and soreness: a systematic and literature review. Sports M edicine, 46, 861-883.

Gámez-Calvo, L., Gamonales, J.M., Silva-O rtiz, A., \& M uñoz-Jiménez, J. (2020). Benefits of hippotherapy in elderly people: scoping review. Journal of Human Sport and Exercise, 17(2),1-12. https:/ / doi.org/ $10.14198 /$ jhse.2022.172.06

Gamonales, J.M. (2020). Fútbol para personas con discapacidad. En Gallado-Vázquez, D., y López-Sa 
las, S. (Editores). Catálogo de investigación joven en Extremadura (Volumen III, pp. 149-152). Cáceres, España: Servicio de Publicaciones de la U niversidad de Extremadura.

Gamonales, J.M., Gil-Sánchez, 0., Porro-Cerrato, C., Gómez-Carmona, C.D., Mancha-Triguero, D., \& Gamonales, F.J. (2018). Psicomotricidad en el aula de Educación Infantil: alumnos con Trastorno de Déficit de Atención e Hiperactividad. Revista Profesional de Investigación, Docencia y Recursos Didácticos, 100(11), 440454.

Gamonales, J.M., León, K., Jiménez, A., \& MuñozJiménez, J. (2019). Indicadores de Rendimiento Deportivo en el Fútbol-7 para personas con parálisis cerebral. Revista Internacional de Medicina y Ciencias de laActividad Física y el Deporte, 19 (74), 309-328. http:/ / doi.org/ 10.15366/ rimcafd2019.74.009

Gamonales, J.M. ., M uñoz-Jiménez, J., Gómez-Carmona, C.D. \& Ibáñez, S.J. (2021). Comparative external workload analysis based on the new functional classification in cerebral palsy football 7-a-side. A full-season study. Research in Sports Medicine, 1-13. https: / / doi.org/ 10.1080/ 15438627.2021.1888105

Gamonales, J.M., M uñoz-J iménez, J., León, K., \& Ibáñez, S.J. (2019). Eficacia del lanzamiento en fútbol para personas ciegas en los Juegos Paralímpicos de 2016. Revista Internacional de M edicina y Ciencias de laActividad Física y del D eporte. 19(70), 361-381. https:/ / doi.org/ 10.15366/ rimcafd2019.76.012

Gamonales, J.M., M uñoz-Jiménez, J., León, K., \& Ibáñez, S.J. (2018). 5-a-side football for individuals with visual impairments: A review of the literature. European Journal of Adapted Physical Activity, 11(1), 1-19. https:/ / doi.org/ 10.5507/ euj.2018.004

Gamonales, J.M., Muñoz-Jiménez, J., M ancha-Triguero, D., \& Ibáñez, S.J. (2020). The influence of the competition phase and the result of match on the competitive demands in football 5-a-side for the visually impaired. International Journal of Performance Analysis in Sport, 1-13. https:/ / doi.org/ 10.1080/ 24748668.2020.1833640

Gorla, J.I., N ogueira, C.D. , Gonçalves, H.R., De Faria, F. R., Buratti, J.R., Nunes, N., Pereira do Rêgo, J.T., Borges, M., Vieira, I. B., \& Labrador Roca, V. (2018). Composición corporal y perfil somatotípico de jugadores brasileños de fútbol siete con Parálisis $\mathrm{Ce}$ rebral de acuerdo con la clasificación funcional. Contribución al Deporte Paralímpico. RETOS: Nuevas Tendencias en Educación Física, Deportes y Recrea- ción, 35(1), 326-328. https:/ / doi.org/ 10.47197/ retos. v0i35. 58931

Guchan, Z., Bayramlar, K., \& Ergun, N. (2017). Determination of the effects of playing soccer on physical fitness in individuals with transtibial amputation. The Journal of Sports M edicine and Physical Fitness, 57 (6), 879-886. https:/ / doi. org/ 10.23736/ S0022-4707.16.06336-2

IFCPF - International Federation of Cerebral Palsy Football. (2020). What is CP Football? Consultado en https:/ / www.ifcpf.com/ (Extraído 22 de noviembre de 2020).

Izzo, R., DeVanna, A., \& Varde'i, C.H. (2018). Data comparison between elite and amateur soccer players by $20 \mathrm{~Hz}$ GPS data collection. Journal of Sports Science, 6, 31-35. https:/ / doi.org/ 10.17265/ 23327839/2018.01.00

Kasiñska, Z., \& Tasiemski, T. (2016). Amputee football in practice and research. Postepy Rehabilitacji, 30(4), 55-66. https: / / doi.org/ 10.1515/ rehab-2015-0055 Kasiñska, Z. , \& Tasiemski, T. (2017). Determinants of sports injuries in amputee football: initial analysis. Trends in Sport Sciences, 24(2), 73-79.

Lozano-Sufrategui, L., Pringle, A., Carless, D., \& McKenna, J. (2017). 'It brings the lads together': a critical exploration of older men's experiences of a weight management programme delivered through a Heal thy Stadia project. Sport in Society, 20(2), 303$315 . \quad$ https:// doi.org/ 10.1080/ 17430437.2016.1173912

Magno, M.P., M orato, M.P., Bilzon, J.L., \& Duarte, E. (2013). Sports injuries in Brazilian blind footballers. International Journal of Sports M edicine, 34(3), 239-243. https:/ / doi.org/ 10.1055/ s-0032-1316358

Mikami, Y., Fukuhara, K., Kawæe, T., Sakamitsu, T., Kamijo, Y., Tajima, H., Kimura, H., \& Adachi, N. (2018). Exercise loading for cardiopulmonary assessment and evaluation of endurance in amputee football players. Journal of Physical Therapy Science, 30(8), 960-965. https:/ / doi.org/ 10.1589/ jpts. 30.960

Mohr, M., Krustrup, P., \& Bangsbo, J. (2003). Match performance of high-standard soccer players with special reference to development of fatigue. Journal of Sports Sciences, 21(7), 519-528.https:/ / doi. org/ $10.1080 / 0264041031000071182$

Montero, I., \& León, 0. (2007). A guide for naming research studies in Psychology. International Journal of Clinical and $\mathrm{H}$ ealth Psychology, 7(3), 847-862.

Peña-González, I., Sarabia, J. M., Mancha-Triguero, D., 
M oya-Ramón, M., \& Gamonales, J.M. (2021). Relationship between physical performance and match load and effects of two consecutive matches in cerebral palsy footballers. RETOS: NuevasTendencias en Educación Física, Deportes y Recreación, 41(3), 728-734. https:/ / doi.org/ 10.47197/ retos. v41i0.86424

Reina, R., Elvira, J.L., Valverde, M., Roldán, A., \& Yanci, J. (2019). Kinematic and kinetic analyses of the vertical jump with and without header as performed by ParaFootballers with Cerebral Palsy. Sports, 7, 1-11.

Reina, R., Iturricastillo, A., Castillo, D., U rban, T., \& Yanci, J. (2019). Activity limitation and match load in parafootballers with cerebral palsy: An approach for evidence-based classification. Scandinavian Journal of Medicine \& Science in Sports. https:/ / doi.org/ 10.1111/ sms. 13583

Reis, R. E. \& M ezzadri, F.M . (2017). Football for people with disabilities and their adaptations in the football country. Revista Brasileira deFutsal eFutebol, 9 (35), 361369.

Silva-O rtiz, A. M., Gamonales, J. M., Gámez-Calvo, L., \& Muñoz-Jiménez, J. (2020). Benefits of inclusive physical activity for people with D own Syndrome: systematic review. Revista Euroamericana de Ciencias Del Deporte, 9(2), 81-94.

Simim, M., Da Mota, G.R., Marocolo, M., Da Silva, B., De Mello, M.T., \& Bradley, P.S. (2018). The Demands of Amputee Soccer Impair Muscular Endurance and Power Indices but $N$ ot M atch Physical Performance. Adapted Physical Activity Quarterly, 35(1), 76-92. https:/ / doi.org/ 10.1123/ apaq. 2016-0147

Simim, M., Silva, B., Marocolo, M., Mendes, E., De Mello, M., \& Da Mota, G. (2013). Anthropometric profile and physical performance characteristic of the Brazilian amputee football (soccer) team. M otriz: Revista de Educaçao Física, 19 (3), 641-648. http:/ / dx. doi.org/ 10.1590/ S1980-65742013000300016 Simim, M.A., Bradley, P.S., Da silva, B.V., Mendes, E.L., De mello, M.T., Marocolo, M., \& Da Mota,
G.R. (2017). The quantification of game-induced muscle fatigue in amputee soccer players. Journal of Sports M edicine and Physical Fitness, 57(6), 766-772. https:/ / doi.org/ 10.23736/ S0022-4707.16.06299-X

Soler, S. (2009). Los procesos de reproducción, resistencia y cambio de las relaciones tradicionales de género en la Educación Física: el caso del fútbol. Cultura y Educación, 21(1), 31-42. https:/ / doi.org/ 10.1174/ 113564009787531253

Tatar, Y., Gercek, N., Ramazanoglu, N., Gulmez, I., U zun, S., Sanli, G., Karagozoglu, C., \& Cotuk, H.B. (2018). Load distribution on the foot and lofstrand crutches of amputee football players. Gait \& Posture, 64, 169-173. https:// doi.org/ 10.1016/ j. gaitpost. 2018.06.008

Thomas, J.R., Nelson, J.K., \& Silverman, S.J. (2015). Research methods in physical activity. H uman Kinetics.

Tweedy, S.M., \& Vanlandewijck, Y.C. (2011). International Paralympic Committee position stand- background and scientific principles of classification in Paralympic sport. British Journal of Sports M edicine, 45(4), 259-269.

Villarejo, D., Belmonte, J.D., Cejudo, A., \& Elvira, J. L. (2019). Efectos de un programa de estiramientos FNP sobre el salto y la flexibilidad en jugadores profesionales de fútbol sala. SPORT-TK: Revista EuroAmericana de Ciencias del D eporte, 8(2), 35-42.

W ieczorek, M., W iliñski, W., Struzik, A., \& Rokita, A. (2015). Hand grip strength vs. sprint effectiveness in amputee soccer players. Journal of Human kinetics, 48(1), 133-139.

Yazicioglu, K., Taskaynatan, M.A., Guzelkucuk, U., \& Tugcu, I. (2007). Effect of playing football (soccer) on balance, strength, and quality of life in unilateral below-knee amputees. American Journal of Physical Medicine \& Rehabilitation, 86(10),800-805. https:/ / doi.org/ 10.1097/ PHM.0b013e318151fc74

Yildiz, S., Kayihan, G., Gelen, E., Cilli, M., \& Ozkan, A. (2018). Acute effects of different warm-up methods on freekick performance in amputee soccer players. M edicina dello Sport, 71(2), 226-233.

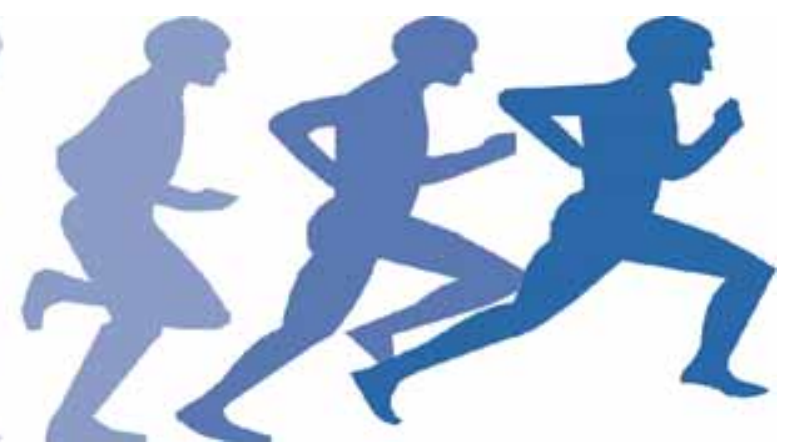

